\title{
Generación inédita. Sentidos de la experiencia liceal de jóvenes que continúan estudiando y son primera generación en educación media en Casavalle, Montevideo
}

\author{
Unprecedented generation. Senses of the high-school experience \\ regarding young people who continue studying and are the first \\ generation in their families accessing secondary education, \\ in Casavalle, Montevideo
}

\section{Paulo Sergio Romero*}

* Educador social (CENFORES, INAU). Licenciado en Psicología (Facultad de Psicología, UdelaR). Magíster en Psicología y Educación (Facultad de Psicología, UdelaR). Diploma «Jóvenes, juventudes y políticas públicas» (Facultad de Ciencias Sociales, UdelaR). Coordinador de la estrategia Centros Promotores de Derechos ( División Socioeducativa, Dirección Nacional de Promoción Sociocultural-MIDES).

* tromeromontero@gmail.com https:/ / orcid.org/ 0000-00034986-8751

RECIBIDO: 1.9.2019

ACEPTADO: 5.11.2019

\section{Resumen}

La reflexión en torno a las desigualdades en educación implica reconocer los disímiles puntos de partida y los diferentes recorridos de los sujetos, en especial en un sistema liceal que desde sus orígenes se ha caracterizado por un mandato selectivo. Para comprender este fenómeno se exponen los sentidos formulados por estudiantes de Casavalle que proceden de familias cuyas trayectorias educativas no han superado educación primaria, y son los primeros en ingresar a secundaria. La progresiva universalización de la educación media requiere saber más sobre los que están arribando, especialmente sobre aquellos que lo hacen por primera vez. En esa línea, es relevante conocer cómo los jóvenes generación inédita componen distintos sentidos, en particular: el contrasentido institucional, las distancias barriales, la importancia de la disponibilidad familiar y el oscilar entre heredar y desheredar. Son múltiples señales que enuncian aspectos sustantivos para la construcción de políticas educativas para todas las personas. Palabras clave: enseñanza secundaria, estudiante de secundaria, integración escolar, Uruguay. 


\begin{abstract}
Reflection on inequalities in education implies recognizing the subjects' uneven starting points as well as understanding their different journeys, particularly in a Secondary Education system that has been characterized by a selective mandate since its origins. In order to grasp this phenomenon, we present the meanings formulated by students from Casavalle, whose families' educational paths have not gone beyond Primary Education and who are the first to enter Secondary Education. The progressive universalization of Secondary Education requires knowing more about those who are arriving, especially those who are first generation in their families. In this regard, it is relevant to understand how the young unprecedented generation compose different meanings, in particular: the institutional contradiction, neighborhood distances, the importance of family availability and the oscillation between inheritance and disinheritance. These are multiple signs that set forth substantive aspects for the construction of educational policies for all people.
\end{abstract}

Keywords: secondary education, secondary school students, school integration, Uruguay.

\title{
Introducción
}

En este artículo se analizan los sentidos que los estudiantes que son primera generación en educación media elaboran sobre la experiencia liceal. Con ese objetivo, es ineludible aproximarse a los orígenes de la educación secundaria uruguaya del siglo XX y su marcado perfil institucional: preparación de las capas altas y medias con vistas a la formación universitaria, para su posterior inserción en los roles directivos de la sociedad (Delio, 2011). Dicha perspectiva permite evidenciar la contraposición entre el mandato de selección de origen y la tendencia al ingreso a educación secundaria perfilada hacia la universalización de la actualidad, que difiere programáticamente de su matriz inicial.

En el contexto uruguayo del siglo XXI, se legisla sobre la educación a partir de la Ley General de Educación (18.437), del 2008, que instituye la obligatoriedad de la educación inicial hasta la enseñanza media superior. En este marco se observa un alto nivel de accesibilidad en los primeros años educativos, que presenta un egreso prácticamente universal en el ciclo primario. Sin embargo, los resultados de la educación media básica y de la media superior continúan siendo insuficientes, en cuanto no se alcanzan los años establecidos como obligatorios para toda la población.

En las últimas décadas se ha incrementado la cobertura de los jóvenes que asisten al sistema educativo, fenómeno que representa un avance en términos de accesibilidad. 
Pero frente a este incremento se observa la fragilidad del vínculo entre algunos estudiantes y la institución educativa. Los derroteros educativos de los jóvenes en Uruguay evidencian profundas desigualdades según los ingresos de los hogares y dichas brechas se acentúan en la educación media superior (INEEd, 2019). En particular, se identifica la incorporación de un mayor número de jóvenes provenientes de familias en situación de pobreza a la educación media, fenómeno que se da con mayor profundidad en las últimas tres décadas. Esto implica una mayor llegada de estudiantes provenientes de familias que no han transitado la educación secundaria, es decir que estas familias recién llegadas no presentan recorridos educativos más allá de los escolares.

En particular, las investigaciones sobre los programas de inclusión educativa destacan este incremento significativo, dado que más de la mitad de los estudiantes que asisten son primera generación en educación media (Mancebo y Monteiro, 2009).

El panorama expresado favorece la discusión sobre la reproducción de las desigualdades sociales por parte del sistema educativo, pero no la explica. En este sentido, establecer una relación directa entre los resultados educativos y el nivel socioeconómico presenta el riesgo de configurar un relato sobre los sujetos referido a las diversas formas del déficit, aspecto que ha influido en los docentes y en la construcción de un tipo de enfoque de políticas educativas que desconfía fuertemente de la capacidad de los jóvenes (Martinis, Míguez, Viscardi y Cristóforo, 2016). Se advierte así sobre las consecuencias de reducir y legitimar la desigualdad de resultados educativos, eximiendo a los actores de la educación, y a las políticas públicas en su conjunto, de las responsabilidades que les competen.

En este sentido, la inequidad expresada en el sistema educativo no es única. Se produce de modo paralelo a otros hitos biográficos, y las brechas en otros aspectos de las trayectorias vitales son más que significativas (Filardo, 2010; De Armas y Retamoso, 2010; Cardozo, 2016). Se evidencian las inequidades establecidas por la estructura económica y social, pero la comprensión de este fenómeno requiere profundizar en otras esferas: en las desligaduras entre los sujetos y las instituciones, en las propuestas pedagógicas, en la vivencia de los jóvenes y en las expectativas familiares; es necesario explorar otras aristas que hacen posible ir más allá del dato. Como señala Karsz (2004), la ruptura o el debilitamiento del lazo educativo se realiza igualmente en lo simbólico y obtiene como resultado una incapacidad para producir sentido y para producir sujetos. En este marco, adquieren relevancia los recorridos inéditos que estos jóvenes transitan, itinerarios que inauguran un vínculo con el liceo, pero que no es solo el de ellos, sino también el de sus familias. 


\section{La linealidad en cuestión: de trayectorias, itinerarios y transiciones}

Las trayectorias educativas son comparables a los juegos de dardos, en los cuales operan los procesos estocásticos. Esto implica una cierta indeterminación descrita por la distribución de probabilidad, pero, incluso si el estado primero es sabido, algunos trayectos pueden ser más factibles y otros no tanto.

La modernidad concibió un esquema en el cual se esperaba de los sujetos una trayectoria normal, en la que se avanza de modo ascendente, cual escalera educativa, con metas pedagógicas fijadas con antelación, a desarrollar en tiempos prescriptos y según los ritmos estipulados como adecuados. Esta visión de la modernidad sustentó la creación de un formato escolar constituido por la homogeneidad del régimen de trabajo, de la regulación de los espacios y de los tiempos de ejecución (Mancebo y Goyeneche, 2010; Tiramonti, 2011).

Los dispositivos de la modernidad fueron claves para lograr constituir un aparato de distribución masiva de la educación, en tiempos en que los Estados-nación y la idea de ciudadano estaban en plena construcción. De este modo, se postulaba una educación que debía ser igual para todos como elemento fundante de la noción de ciudadanía. No obstante, ya a fines del siglo XX comenzó a vislumbrarse la paradoja que este modelo implicaba en términos de reproducción de desigualdades sociales. Estos aspectos promovidos por las instituciones de la modernidad asignaron modos de ser y hacer que, en el contexto actual, quedan interpelados al verificarse las diversas y desiguales trayectorias que los sujetos transitan. Para ampliar la comprensión de ello son fundamentales los aportes sobre los prototipos de trayectorias que desarrolla Terigi (2009). En este marco, la autora describe el modelo de trayectoria teórica, que ha estructurado históricamente el saber pedagógico, en contraste con las trayectorias reales, que son aquellas que efectivamente realizan los sujetos, trascendiendo la norma establecida como esperable y deseable. Esta tensión es observada en la escena liceal de modo incesante, al colisionar las expectativas sobre los estudiantes con los itinerarios que estos construyen en su tránsito por el sistema educativo.

Para adentrarnos en el debate en torno a las trayectorias educativas, es pertinente incorporar el análisis sobre las transiciones vitales (Casal, 1996; Casal, García, Merino y Quesada, 2006; Stauber y Walther, 2001) «que analiza los cambios de estados que procesan los individuos a lo largo del ciclo vital» (Filardo, 2010, p. 6). En tal sentido, en las transiciones contemporáneas se identifica una diversidad de secuencias: de las lineales a las intercaladas; de los itinerarios de emancipación relativa a los truncos o inestables; de las progresivas a las recursivas o de vaivén. Por tanto, en la coyuntura actual se pone de manifiesto que las cualidades de estos hitos vitales son variables, al igual que las 
trayectorias educativas, y en ambos casos están en juego otros aspectos: los subjetivos, del género, de la etnia-raza, del entorno familiar y del contexto sociohistórico. Las transiciones de hoy representan nuevas lógicas subjetivas. Estos hallazgos provenientes del campo de las transiciones amplían la perspectiva en torno a las trayectorias educativas, a la vez que permiten evidenciar el proceso de erosión de las instituciones para regular la vida social y producir una subjetividad única y compartida, si es que alguna vez existió.

En la sociedad contemporánea, el concepto de individualización es una noción clave que implica el pasaje de la identidad como dato a la identidad como tarea. En el sujeto, refiere a las biografías «hágalo usted mismo» (Beck y Beck-Gernsheim, 2003, p. 47), lo que conlleva que sean los jóvenes quienes construyen su propio itinerario, sin depender de un contexto estable. Por tanto, la individualización tiene efectos subjetivos: «cuando la transición a la vida adulta varía de la normalidad a la incertidumbre, los jóvenes sufren una presión cada vez mayor» (Du Bois-Reymond y López Blasco, 2004, p. 16). En el sentido expresado, y llevado al ámbito educativo, es posible identificar la recarga individual, mientras el estudiante se encuentra obligado a responsabilizarse por las consecuencias de su biografía. En este esquema, es el sujeto quien asume todo el riesgo, dado que entre el estudiante y la institución no hay lazo. Desde el análisis biográfico por itinerarios vitales, se considera que el sistema de transiciones es sociohistórico y geopolítico, y estos factores inciden en la configuración de los itinerarios (Casal et al., 2006).

Este modo de comprender los itinerarios es diferente al que alude a una racionalidad basada exclusivamente en las decisiones individuales. En este marco, el concepto de itinerario puede ser un concepto hermenéutico, en la medida en que alude a la orientación y descripción de un determinado trayecto y se lo puede definir como el recorrido que incluye los avances, los descansos y los accidentes, a la vez que incorpora los regresos que pueden darse en el recorrido educativo, quebrando la idea hegemónica de linealidad. En este sentido, la posición que una institución o sistema educativo tenga sobre las trayectorias educativas es de relevancia y requiere ubicar las tensiones existentes entre los aspectos singulares, institucionales y sociales. Esto es de gran importancia para analizar los diversos derroteros que siguen los sujetos, entre el determinismo y el antidestino (Núñez, 1999), especialmente cuando es una primera generación.

\section{Antecedentes relevantes y abordaje metodológico}

La progresiva universalización de la educación media requiere saber más sobre los que están arribando por primera vez. El debate y las investigaciones centradas en las trayectorias educativas han brindado como resultado una serie de documentos que evidencian la problemática de la educación media (Terigi, 2009; Fernández, 2010; De 
Armas y Retamoso, 2010; Filardo y Mancebo, 2013). No obstante, las investigaciones señalan la pertinencia de profundizar en las experiencias de los estudiantes e incorporar la perspectiva de los jóvenes.

En el marco de la investigación social, se configuran como antecedentes sustantivos diversos estudios que examinan la relación entre juventud y educación (Saraví, 2009; Filardo, 2010; Núñez, 2010; Acosta, 2011; Di Leo, 2011, Viscardi y Alonso, 2013); entre culturas juveniles y culturas escolares (Dubet y Martuccelli, 1996; Kessler, 2002; Dubet 2011; Weiss, 2012); también los centrados en los estudiantes, docentes y propuestas educativas (Bordoli, 2007; Filardo y Mancebo, 2013; Vaccotti, 2014; Ruiz Barbot et al., 2014) y aquellos que recogen los sentidos y representaciones de los estudiantes sobre la educación media (Espíndola, 2007; Ventós, 2015; Dibot, 2015). De modo complementario, son fundamentales las investigaciones que exploran la relación entre sujetos, educación y desigualdades (Baquero, 2001; Duschatzky y Corea, 2002; Tenti Fanfani, 2007; Tiramonti et al., 2008; Dussel, 2009; Skliar, 2011; Zelmanovich y Minnicelli, 2012; Martinis, 2013; Ruiz Barbot, 2015).

Para profundizar en los aspectos singulares de este artículo, han sido de relevancia las investigaciones referidas a la segregación y exclusión territorial (Wacquant, 2001, 2010; Merklen, 2005; Aguiar, 2009) y, en particular, los antecedentes de investigaciones que tienen como escenario la cuenca de Casavalle en el contexto uruguayo (Filardo, 2005; Espíndola, 2007; Folgar y Romano, 2007; Álvarez Pedrosian, 2013). Por último, en referencia al objeto de estudio generación inédita, no se encontraron antecedentes en el contexto nacional. Sí se identificaron los aportes desarrollados por Foglino, Falconi y López Molina (2009) en Argentina, los cuales conectan en forma directa con el fenómeno explorado y se han convertido en una referencia ineludible.

Este repertorio de investigaciones indica que las desigualdades se reproducen en los centros educativos y expresan una sumatoria de exclusiones, aspectos que son primordiales para comprender la arista seleccionada. Dichos antecedentes permiten desarrollar una visión integral, incorporar conceptos, discusiones y datos que han sido necesarios para comprender el vínculo entre los sujetos primera generación, sus familias y la institución educativa, en un contexto de desigualdades.

Se diseñó una investigación de carácter cualitativo que estudió las experiencias liceales desde la perspectiva de los estudiantes. Se buscó analizar sus puntos de vista, partiendo de los individuos, de sus relatos y de sus actividades. El universo de estudio comprendió a jóvenes (mujeres y varones) de 13 a 18 años, pertenecientes a familias que no han transitado por el liceo.

El muestreo teórico para la investigación se realizó a partir de un muestreo de tipo intencional. Como criterio general, se definió que fueran jóvenes residentes en la cuenca de Casavalle (Montevideo) durante 2016-2017 y que tuvieran al menos un año de experiencia liceal. En todos los casos seleccionados los jóvenes se encontraban estudiando 
en educación media, es decir, presentaban continuidad educativa en diversas ofertas de enseñanza en UTU, liceos o programas de inclusión educativa, ya que la investigación se centró en la experiencia liceal y no en la desvinculación educativa.

La fuente de información fue primaria, a partir de entrevistas a los jóvenes seleccionados, donde se incorporaron preguntas y estímulos narrativos para la recolección de datos biográficos respecto a cierto problema social (Flick, 2004); en este caso la pauta de entrevista se centró en los sentidos de la experiencia liceal y los significados de ser estudiante. A su vez, se preguntó sobre las expectativas que tienen sus familiares sobre la educación en general y sobre ellos en particular. Para la implementación se determinó un número mínimo de entrevistas a realizar, que se revisó a partir del criterio de saturación teórica propuesto por Glaser y Strauss (1967). Así, se realizaron en total 16 entrevistas (dos de ellas fueron pre-test).

Se definió esta zona de Montevideo porque es un caso paradigmático de fragmentación social. En este territorio es factible encontrar a la población objetivo de la investigación, y al mismo tiempo el espacio se caracteriza por el predominio de un nivel educativo máximo en la educación primaria. Además, presenta altos índices de población joven que tiene un frágil vínculo con las instituciones de educación media (Filardo, 2005; Lombardo, 2005; Espíndola, 2009). Para la documentación de datos se realizó un registro con un enfoque narrativo-biográfico que incluyó la producción de relato mediante dos técnicas de investigación cualitativa: entrevistas individuales y trayectos educativos con producción de soporte gráfico.

Al inicio de la entrevista se invitaba a los jóvenes a construir su trayecto educativo, tomando los hitos de su experiencia liceal como eje de la producción. Se consideró el recorrido educativo y social, además de la trayectoria curricular, ya que el trayecto habla de un camino que se va haciendo y un caminante que es quien lo hace, como señala Ardoino (2005). El trayecto implica para el sujeto dar cuenta de un tiempo transitado, en el que hay cambios, alteraciones y la presencia de otros que influyen en ese andar. En referencia al análisis cualitativo del contenido, se incorporó la perspectiva de los actores sociales en su vida cotidiana mediante una descripción detallada de la realidad (Denzin y Lincoln, 1994), considerando que las interpretaciones cualitativas surgen de un proceso de construcción, que en este caso fue el intercambio con los estudiantes. Se identificaron los temas emergentes $y$, a partir de ello, se fueron construyendo las dimensiones analíticas con la intención de comprender los sentidos atribuidos a la experiencia liceal. Para el análisis de la información se definió un recorrido que comenzó con una lectura sincrónica y diacrónica de las entrevistas, la construcción de dimensiones analíticas, la codificación de la información y el análisis transversal de los relatos de los jóvenes primera generación. En todo el proceso se consideraron como criterios de fundamentación la verosimilitud del procedimiento y su documentación, la validez de los datos y la transferibilidad (Guba y Lincoln citados por Pla, 1999) y su triangulación (Flick, 
2004). A partir de las consideraciones explicitadas se desarrollaron las principales líneas de análisis que en este artículo se resumen.

\section{Sentidos de la experiencia liceal}

En el marco de la investigación «Generación inédita» (Romero, 2018), los jóvenes narran y manifiestan que al comenzar al liceo deben enfrentar una experiencia nueva, que los desafía a resolver una primera situación: la transición del ámbito escolar al liceal. Ellos dan cuenta de una experiencia con un sentido novedoso, que es tanto de profundos cambios en la subjetividad como de traslados geográficos. Es un pasaje que conlleva movimientos internos que identifican con sentimientos de ansiedad, miedos y curiosidad, y también de crecimiento. Por tanto, es una experiencia de cambio sustancial a la que se enfrentan con ganas, incomodidad y desencaje. Hasta aquí, la experiencia no es tan distinta a la de otros jóvenes que transitan el mismo pasaje. Entonces: ¿cuáles serían las particularidades de este tránsito en los estudiantes primera generación?; ¿qué es lo nuevo en ellos?; ¿cómo resuelven la ausencia del legado, en cuanto sus familias no podían referir a sus propias experiencias liceales? Frente a estas interrogantes, los jóvenes advierten que las maestras, los hermanos y los compañeros del barrio que fueron al liceo son quienes transmiten la experiencia liceal. Son figuras mediadoras que desempeñan un rol fundante en la conexión con ese nuevo espacio a explorar. Son quienes anticipan lo que está por suceder, ayudan a componer el sentido de lo liceal. En este marco, las madres y padres ${ }^{1}$ tienen un papel central en la experiencia liceal, son quienes impulsan a los jóvenes. Aunque no puedan transmitir la experiencia, dado que no la han vivido, desarrollan la función subjetivante de acompañar el proceso educativo de sus hijos e hijas, y ello es reconocido como sustancial. Es decir, los jóvenes manifiestan que el apuntalamiento familiar es constitutivo para el ingreso al liceo.

El sistema educativo recibe año a año a las nuevas generaciones, pero en gran parte desconoce los recorridos previos (el de los sujetos y sus familias). En este pasaje se origina una experiencia contrasentido: novedad en los sujetos y recurrencia en la institución. Sin embargo, este recorrido, que es inicialmente similar, no tiene iguales consecuencias para todas las personas, ya que para algunas se convierte en una experiencia inédita y trunca, de modo tal que este pasaje no es armonioso y tiene costos. En particular, para un grupo de estudiantes es un primer cimbronazo que desestabiliza y quiere ser evitado, en especial cuando los deja afuera.

Esta descripción es válida para los relatos de los jóvenes que tienen madre o padre, convivan o no con ellos; cuando estos están ausentes, otras figuras emergen para cumplir el rol de referentes: abuelas, tías y hermanos. 
Es posible afirmar que el liceo es importante para estos jóvenes, ya que así lo expresan. Los estudiantes señalan que perciben cambios respecto a la escuela, en particular en el encuentro con el mundo adulto. Remarcan las diferencias entre los formatos de aprendizaje, el pasaje de un sujeto enseñante a una multiplicidad de profesores; manifiestan que en el ámbito escolar la maestra inviste el orden explicador - es quien organiza y da sentido, es una figura singularizada-, pero que esa modalidad vincular en el liceo se difumina. En la evocación de la escuela establecen un sentido de cercanía con la institución y sus maestras; las recuerdan en un lugar positivo, y esa vivencia es unívoca. Esto dista del modo en que relatan el vínculo con los profesores, los cuales son evocados con sentidos polivalentes. Para ellos, sus profesores son significativos, pero los representan con una modalidad relacional variada, pendular: desde aquellos que enseñan a aquellos a los que nada les importa, desde aquellos que los apoyan hasta quienes se enojan. Estas miradas dan cuenta de la diversidad en los modos de encarnar la docencia y sus resonancias en el espacio liceal y, en consecuencia, en las subjetividades de los jóvenes. De tal modo, algunos estudiantes devuelven y replican los modos vinculares que ofrecen los adultos en el liceo y establecen una relación reflejo.

Los docentes son recuperados en los relatos en un lugar de privilegio: importan, dejan huellas de las fructíferas y de las inconclusas, en las que no logran alcanzar sus expectativas de enseñanza. En este sentido, se identifican trayectorias educativas truncas en algunos de los jóvenes primera generación; aquellas sin entendimiento, en la que se tensionan el modelo institucional, el lugar docente y el ser estudiante. En esta línea, expresan los efectos de una relación en ausencia, confusa, en la que se claudican las funciones del enseñar: «me trataban bien, pero no me iba bien»; «son rebuenos... y como que no nos exigen». En este sentido, se observa la repercusión que tienen los proyectos pedagógicos en los sujetos y sus narrativas. Por ello, es significativo subrayar que los jóvenes reconocen desde un lugar relevante a aquellos docentes que logran acompañar, que logran sincronizar con sus tiempos de aprendizajes. Este modelo de profesor, que logra escuchar y sintonizar, es central porque entiende la diferencia entre ser lento y aprender lento; tiene más de un modo de enseñar y está dispuesto al encuentro; es el que ellos recuperan en su memoria, porque tiene constancia y sabe de la importancia de la cronicidad, de la regulación necesaria para entablar el vínculo educativo.

Se vuelve evidente, como señala Dubet (2011), que no es posible analizar la experiencia educativa de los estudiantes sin analizar la relación pedagógica. De tal modo, si no hubieran existido las operaciones de entender, de saber, de aprender - la vivencia del «me explicaba a mí, al lado mío» o la del «empezó a hablar despacio y ahí empecé a entender»-, la experiencia liceal no hubiera prosperado. Los jóvenes recuperan 
escenas y anécdotas en las cuales los adscriptos tienen un lugar significativo. Identifican y dan relevancia a su figura dentro del ámbito liceal, los ubican como referentes en la institución y los nombran en el vínculo con el liceo. Son mediadores, dan significado intersubjetivo a las acciones, en ellos los jóvenes se sienten interpretados y acompañados - «hablamos con la adscripta»— , son quienes interceden en el conflicto. Frente a lo cotidiano y lo excepcional de la vida liceal, se los describe como los que saben, los que se contactan con ellos y sus familias, y los reconocen. Este último término es sustancial en la medida en que los sujetos configuran sus vínculos educativos en torno al reconocimiento, o en la ausencia de este. Se componen en una figura nueva e importante para ellos, y su tarea adquiere mayor relevancia porque son los que permanecen, los que interfieren y articulan el espacio educativo. El sentido que los jóvenes primera generación formulan sobre los adscriptos no es específico, sino una vivencia que también comparten otros estudiantes. No obstante, en sus relatos son figuras significativas, y en consideración de lo inédito de su experiencia son traductores con los que cuentan al circular por el espacio liceal.

\section{Experiencia de aprender, o la nada}

Cuando se indaga sobre qué impulsa a los jóvenes hacia la experiencia liceal, se identifica que en todos los casos el interés por aprender se mantiene como un sentido inicial. Es decir, quieren ir al liceo para saber más, pero ello no siempre se logra sostener. Describen con claridad el deber ser del estudiante, categóricamente identifican los requerimientos de la vida estudiantil y reflejan el conocimiento sobre su significado: eso lo saben. Exponen con certeza las competencias que se requieren y expresan que se han sentido así; dan cuentan del oficio y reiteran una serie de cualidades para describirlo: «ser estudiantes es ir al liceo, estudiar para las materias»; «tipo que faltás a veces al liceo, pero casi todos los días vas»; «que tenés las materias altas, no les faltás el respeto a los profesores»; «un estudiante va a ir a un lugar que le van a enseñar y tiene que prestar atención».

Una parte de los jóvenes identifican la experiencia de saber, que da cuenta del conocimiento sobre para qué se estudia. Ello se expresa en los sujetos que tienen una vivencia satisfactoria de pasaje por el liceo, que es acompañada de la posibilidad de identificarse a nivel vocacional con algunos referentes. Así, profesores, amigos, primos, jefes son quienes ellos reconocen para imaginar su futuro educativo. Establecen en todo momento que el apoyo familiar es fundamental para alcanzarlo. Los relatos expresan búsquedas de vocación, existe un quiero ser: «fotógrafa», «contador», «escultor», «peluquera», «profesor de educación física», «cocinera», «psicóloga», «electricista», etc. A partir de sus experiencias, van ideando un proyecto que les interesaría desarrollar y que 
logran nombrar. El saber de la experiencia es un saber paradójico (Contreras-Domingo, 2013), y en los jóvenes se observa que sus vivencias son las que posibilitan encontrar una dirección. Se evidencia el lugar central del quehacer educativo en el asumir la tarea de acompañar esa orientación y ofrecer nuevas preguntas.

Por lo expresado, el liceo para algunos jóvenes generación inédita tiene un sentido vinculado al conocimiento y la proyección de una vocación, en la medida en que se encuentran con un otro disponible con quien el estudiante puede identificarse, en un contexto de apuntalamiento familiar. Pero para un segundo grupo de jóvenes, se formula un sentido entradasalida sobre la experiencia liceal, lo que no implica necesariamente salirse del sistema educativo, pero sí de los liceos. Aquí, los jóvenes señalan el «no entender», lo cual se manifiesta como un problema primordial. Es una expresión que se enuncia centralmente en sus narrativas y está asociada al significante «nada». El «no se me pegaba nada», que los jóvenes relatan, refiere a la experiencia liceal sin relación, sin posibilidad de vínculo que permita adherirse a lo educativo. De tal modo, el liceo se convierte en un espacio inocuo, que configura una expresión más de la nada, de aquello que no ocurre para ese sujeto; es un estar sin acontecimiento, en falta de sentido. Por tanto, es posible identificar que la propuesta pedagógica se vuelve inaccesible para estos jóvenes. Las narrativas exponen la discusión establecida en el campo pedagógico y social, en torno a las posibilidades de educabilidad de todos los sujetos; pareciese que la certeza sobre la condición crítica y de subsistencia de algunos jóvenes se desliza, como expresa Baquero (2001), a una certeza sobre la imposibilidad de una acción educativa próspera. Este enfoque inhabilitador a priori se identifica en los centros educativos a través de expresiones que los sujetos internalizan y de las cuales se sienten responsables: «[a mí] no se me pegaba nada». Del mismo modo, esta vivencia es incorporada por las familias, al no optar por la continuidad en la institución liceal.

Los jóvenes manifiestan que, luego de desvincularse de los liceos, se orientan hacia otras propuestas o formatos pedagógicos en los que sienten que pueden. Estos casos se dan entre los más jóvenes, que luego de haber repetido un año o dos se van a la UTU, a experiencias de Formación Profesional Básica (FPB- FPB/C) o programas de inclusión como Aulas Comunitarias. Estas biografías entradasalida del liceo evidencian la confrontación de un modelo considerado para ser transitado por un prototipo de estudiante, pero en el que participa una gran variedad de sujetos pedagógicos. Quienes no logran cumplir con la expectativa de normalidad trazada por la institucionalidad liceal quedan excluidos. Entonces, cuando la experiencia de no entender se profundiza, emerge la nada, la desligadura subjetiva, la falta de sentido, aquello que los hace sentir afuera. Experimentan la falta de entendimiento, estar en el liceo no es válido para ellos - que es muy distinto a creer que no tiene valor-. Desde esta perspectiva, la opción de no estudiar no es para todos, sino especialmente para algunos que habitan los barrios más vulnerados. 
En las narrativas se expresa, como si fuera una condena natural, que los que dejan de estudiar terminan en la esquina. Se salen del camino, es decir, comienzan a habitar lugares distintos en el espacio social: «a los que no van al liceo casi nunca me los cruzo». Se establecen así las diferencias entre los que estudian y los que no, y se evidencian las distancias y brechas con los jóvenes «de la esquina», los que no estudian. Como expresa Feixa (2007), el estar dentro o fuera de la institución educativa trae efectos, y esta es una tensión histórica. Por ello, la centralidad de encontrarse insertos, aprendiendo, en este tiempo vital es indiscutible. Los itinerarios ratifican que el marco de la ley no llega para todos por igual. El estar afuera, ser parte de la esquina, el hacer la nada, da cuenta de «un aflojamiento de lazo social que habría marcado la ruptura de los individuos respecto de sus inserciones sociales [...]» (Castel, 2004, p. 63). Pero estas desigualdades se vuelven obscenas cuando para algunos jóvenes el no estudiar no es una opción, ni siquiera se imaginan no estudiando, no tienen elaborado ese estatuto en su subjetividad, mientras que para otros es una experiencia cierta, que llega cuando la nada acontece. De tal modo, el liceo despliega, una vez más, su mandato de origen vinculado a la selección, y con un sentido lógico entabla la expulsión de algunos. El análisis da señales de dos tipos de experiencias liceales que se les ofrecen a los jóvenes en nuestra sociedad: una para los que saben y otra para lo que no saben (estar dentro del liceo). Los itinerarios advierten el imbricado vínculo entre los saberes, las explicaciones y los entendimientos, y se sintetiza entre el aprender o el no aprender. Por ello, emerge la necesidad de revisar, de desmantelar la oferta única, la propuesta pedagógica que la institución habilita, valoriza y recompensa.

\section{El futuro bien}

Los jóvenes generación inédita señalan que los estudios son garantes de mejores trabajos y tienen la convicción de que es posible obtener un futuro promisorio a partir de los avances en el ámbito educativo. Esta certeza narrada no solo se encuentra en ellos, sino que también está enraizada en las palabras de sus familias. Seguir estudiando para tener un «futuro bien» puede interpretarse en alusión al porvenir, originado en los estudios y alcanzado en el trabajo. Expresa un significado presente en pos de adquirir un patrimonio: simbólico, económico, educativo...

Los planteos que los jóvenes generación inédita efectúan coinciden con las representaciones descritas, por cuanto ellos identifican que a través de sus esfuerzos educativos individuales es factible su inserción en el mercado laboral. En sus narraciones afirman la importancia de los estudios para obtener un buen trabajo, y expresan que para alcanzarlo deben seguir estudiando. Esta visión coincide con los planteos de Kessler (2007) y Dubet (2011), que identifican una concepción instrumentalista de la 
educación. Es decir, para una parte de los jóvenes la formación es vista como medio para acceder al mercado laboral, y llega a establecerse como un período a la espera de ese acceso.

Entre los hitos vinculados al mundo del trabajo, estos jóvenes que aún estudian establecen frente a la experiencia liceal un sentido que se representa en segmentos, en el entendido de que la culminación del primer ciclo y la llegada a la mayoría de edad son punto de inflexión, de quiebre dentro de sus biografías educativas. Consideran que lo que no se logre en referencia a los estudios hasta ese momento no se alcanzará después. Se representa así un tiempo de fin de los estudios que con frecuencia se identifica como el umbral de lo educativo y con la certeza del comienzo de un nuevo tiempo: el de trabajar, si ya no se empezó antes. Entonces, ¿para algunos jóvenes el futuro es más cercano?, ¿existe menos tiempo para entrenarse o preparase para el mundo adulto? Y, en consecuencia, ¿se acota el tiempo de experimentar y habitar el oficio de estudiante?

La noción de moratoria social permite identificar el plazo concedido a ciertos jóvenes; un tiempo con menores exigencias mientras se culminan los estudios, hasta que logren cierta autonomía social y económica. Por tanto, es una posibilidad de ampliar el período de aprendizaje y, por ende, de ser estudiante. Esta condición es la que la mayoría de los jóvenes entrevistados no imaginan disfrutando, más allá de los tramos educativos iniciales (ciclo básico). La discusión en el campo social en torno a la moratoria se fue profundizando a partir de concebir las diferencias entre los sujetos según las condiciones económicas y observarse que no todos los jóvenes acceden a este tiempo, a este entre que les permite usufructuar su condición de estudiantes. En los relatos, la representación que traen los generación inédita en torno a los estudios y sus incompatibilidades evidencia la imposibilidad de inscribirse en ese privilegio de prolongar su pasaje al mundo adulto. Se expresa que es irreconciliable la idea de ser mayor de edad y estudiar, o la idea de ser padres y estar dentro del sistema educativo. Se los representan como tiempos vitales diferenciados, que no conviven, que no son acordes.

El enfoque de moratoria social implica el riesgo de reproducir un modo dominante de ubicar lo juvenil y de dejar por fuera a una diversidad de jóvenes a los cuales no se les concede ese plazo, a quienes se describe como jóvenes no juveniles, como señala Urresti (2001). Por lo expresado, es fundamental incluir los riesgos mencionados, que permiten comprender los desiguales acuerdos que se les ofrecen a los jóvenes según los sectores sociales. Mientras tanto, estas brechas en lo consensuado según el grupo social de pertenencia contribuyen a la fragmentación y protegen la reproducción social. El nudo en torno a las perspectivas del futuro y las búsquedas por evitar la reproducción de la trayectoria educativa surgen de modo reiterado. Expresan una subjetividad que balancea entre el decir de ellos - los estudiantes- y el decir de sus familias, hasta el punto de confundirse en algunos relatos, pues de modo constante describen que para sus familias el hecho de que asistan al liceo es beneficioso y se asocia al futuro. Esto es 
así, particularmente, porque en el caso de ellos encarna la posibilidad de construir un itinerario nuevo, inédito, que no reproduce las historias educativas de su entorno.

En este marco, la reproducción del discurso social y/ o familiar sobre el futuro encuentra asidero en ellos. Sobre este aspecto Ruiz Barbot et al. identifican que «los y las adolescentes incorporan este discurso parental y lo resignifican» (2014, p. 58). Indican que, para los jóvenes que viven en barrios con menores soportes económicos, la educación posibilitaría un lugar, en tanto los aleja de la experiencia laboral y educativa parental y les permite establecer distancias de su entorno. La educación portaría el sentido de pasar a existir socialmente, «ser alguien en la vida», a diferencia de aquellos adolescentes de otros sectores que vinculan la educación al proceso formativo, en el presente.

A diferencia de lo que acontece en otras familias donde se trabaja para dejar huellas de la tradición educativa, formativa y profesional, las familias de estos jóvenes se esfuerzan, y fuerzan a que sus hijos no reiteren sus derroteros educativos y, por efecto, sus trayectos ocupacionales. En estas circunstancias el esfuerzo subjetivo para el joven pulsa entre el sentido de heredar-desheredar. De este modo, el análisis coincide con los estudios que expresan esta vertiente (Bourdieu y Passeron, 1964; Dubet, 1996; Falconi, Foglino y López, 2008; de Gaulejac, 2013). Sin embargo, la continuidad educativa no está garantizada para la mayoría de estos jóvenes, como se expresó, y la experiencia liceal comienza a erosionarse en la medida en que los estudios no conectan con las demandas vitales. Así, la educación en una sociedad desigual es un patrimonio en disputa, donde algunos educan para eternizar la herencia y otros para generar cambios, paradoja que sintetiza, en parte, las contradicciones de un sistema educativo fragmentado.

\section{Familias e itinerarios}

En esta dimensión los jóvenes relatan sentirse acompañados por sus referentes familiares y se diferencian de aquellas familias del barrio que no apoyan a sus hijos o nietos. La experiencia de apoyo es un elemento común en las entrevistas y ocupa un lugar fundamental en sus relatos. ${ }^{3}$ En referencia a la composición familiar, la situación es diversa: hay familias nucleares, con jefatura femenina, y otras ensambladas; estas últimas son la mayoría. En particular, es significativo el rol de las abuelas, que en algunas historias son figuras vertebradoras que impulsan a ir al liceo. En sus narrativas los jóvenes expresan que las familias inciden sobre sus procesos educativos, aspectos que se manifiestan en anécdotas y situaciones de la vida cotidiana. Son hacedoras de acciones

Es importante considerar que las entrevistas se realizan en centros juveniles de Casavalle que funcionan complementando las actividades de educación formal, por lo cual es posible inferir que esta característica expresa que estas familias abogan por la presencia de los jóvenes en los ámbitos educativos. 
- que se traducen en aliento e insistencia-, de gestos que van de los diálogos a las mediaciones, del preguntar al empuje matinal. Perciben a diario en sus familiares el acompañamiento de diversas formas. Los gestos familiares relatados se describen como actos corrientes en los que se abordan los temas de estudio y se habla sobre el proceso educativo. Se vinculan a un tiempo y espacio especial, que se da en el acompañar rutinas y en el impulsar a seguir estudiando.

Pero es posible identificar un tipo de diálogo particular que es revelador y que los jóvenes evocan en modo significativo. Son aquellos intercambios que usualmente se dan en momentos cruciales en la trayectoria educativa: en el pasaje de ciclo, frente a situaciones de repetición escolar, frente a una notificación del liceo o cuando se produce un cambio de rumbo (generalmente en el pasaje del liceo a la UTU). Son diálogos entre crisis, que mezclan diversos sentidos: a veces apuntalan a los sujetos en su continuidad educativa, en otras ocasiones los culpabilizan, y en algunos casos traen recuerdos, evocan sus historias personales. Son los diálogos que balizan el itinerario, que se configuran en enunciados subjetivantes que permiten a los jóvenes esclarecerse, escucharse y construirse en un marco vinculante entre sus historias, las de la familia y el liceo. Así, los diálogos inscriben en lo simbólico; es la posibilidad de hacer espacio en la medida en que hay un otro disponible que permite su despliegue. Es decir, los jóvenes recuperan en sus relatos estas conversaciones donde aparece el límite, se acompaña el deseo o se insiste en poner en palabras lo acontecido. En estas experiencias de primera generación, los jóvenes señalan que para sus familias los estudios son importantes. De tal modo, perciben y relatan el peso en caso de cumplir, o no, con las expectativas trazadas por el mundo adulto. Al respecto, el proyecto parental se identifica como impulso que condiciona la trayectoria, y es sustancial por cuanto la proyecta o, por el contrario, la limita. Como señala de Gaulejac (2013), refiere al conjunto de representaciones que los padres se hacen del futuro de sus hijos, de modo consciente e inconsciente, en un contexto social que favorece o impide su realización. Los referentes familiares son evocados durante las entrevistas en diversidad de situaciones, donde se pone en juego el proyecto parental y emerge el correlato comparativo entre las historias de los estudiantes y la de sus familias.

Como explicita Bleichmar (2003), la trama generacional de la historia familiar se vuelve a editar en cada nueva etapa, a partir de las relaciones que se establecen con los hijos, en tanto los padres desempolvan, recuperan y actualizan sus experiencias vividas con sus padres. Sobre ello se exploraron las elaboraciones que los jóvenes realizaban sobre sus referentes familiares, en particular la pregunta de por qué ellos no habían ido al liceo. En los relatos se caracterizan algunas respuestas, como aquellas vinculadas a las instituciones (por ejemplo, la crianza en el Consejo del Niño), las que remiten a hechos históricos (específicamente a la dictadura) y otras elucubraciones que remiten al tenor de época: explican que antes se comenzaba a trabajar tempranamente y «no se 
necesitaban los estudios como hoy en día», o señalan que las madres no estudiaron porque fueron relegadas al espacio intrafamiliar en vinculación con los cuidados. Es así que los diálogos familiares expresan las imposibilidades e incompatibilidades de las generaciones anteriores para continuar los estudios y señalan el interés de que estos jóvenes no reproduzcan sus trayectorias educativas. Como indican Bearteux (1994) y de Gaulejac (2013), a través de las historias familiares es posible comprender los procesos de formación de la trayectoria social que han recorrido, estableciendo su carácter sociohistórico.

En su memoria, los jóvenes rescatan las historias y el periplo familiar que les han contado. Se describen escenas que van del orgullo al desencanto, en tanto los estudiantes experimentan una serie de contradicciones al hallarse tironeados entre las aspiraciones colectivas de su lugar de pertenencia y las aspiraciones individuales. Como señala de Gaulejac, «los hijos pueden sentir la deuda por el esfuerzo hacia sus padres» (2013, p. 52). Asimismo, Falconi plantea que «distinguirse de los padres, superarlos y, en un sentido, negarlos, no está exento de un alto costo subjetivo» (2009, p. 9). En las historias emergen con nitidez estas vivencias ambivalentes, al igual que otros sentimientos desencontrados, especialmente cuando no se alcanza a cubrir las expectativas familiares.

No obstante, también se identifican otros tipos de historias, las que recompensan y superan a la generación de sus padres. Esta construcción de los primeros en llegar carga con el orgullo y el peso de diferenciarse, de ser lo que sus padres no fueron, que les demanda convertirse en aquello que sus padres no pudieron ser. En este último sentido, los jóvenes saben cuál es la distinción, por dónde se expresan las diferencias. Uno de los relatos paradigmáticos identifica este sentido del diferenciarse: «me hacía la linda hablando con palabras raras». Estas palabras refieren a las aprendidas en el liceo, que son distintas a las que se utilizan en su casa, y esta acción manifiesta en parte las distancias con su entorno, a partir de lo adquirido en el espacio liceal. Nuevas palabras que dan cuenta de la apropiación de la experiencia inédita, en conocimiento del valor que tiene saber y contar con más palabras, hasta el punto de expresar la percepción de la superación generacional: «yo sé hablar mejor que vos». Se formula así el sentido sobre el liceo palabra, lugar donde se aprenden otras formas de hablar e intercambiar con lo distinto.

Los múltiples sentidos que los jóvenes relatan dan cuenta de que para ellos sostener los estudios no es algo obvio, porque no son estudiantes herederos de una tradición familiar vinculada a lo educativo. Como señala Dubet (2011), estos jóvenes se diferencian de aquellos para los cuales el acceso a la educación media se da de un modo natural y los estudios se viven como evidentes por la continuidad y la histórica vinculación entre su cultura familiar y la cultura escolar. Las diferencias sociales se cristalizan de diversas formas, y el modo de hablar es una de la que los jóvenes primera generación recuperan. La distribución de las palabras, al igual que otros bienes - patrimoniales, culturales- 
no llega a todos por igual, y eso se reconoce, se sabe con qué cantidad de palabras se cuenta y qué valor tiene socialmente.

Dentro de la investigación se exploró acerca de los modos en que los jóvenes y sus familias resuelven los problemas académicos que la vida liceal plantea, en un marco sin antecedentes en educación media. Es decir, ¿cómo logran, o no, compensar, ayudarlos, delegar, cuando los requerimientos del liceo exceden sus posibilidades? De los relatos surgen ciertas escenas de mediación con el conocimiento, frente a lo inédito de lo liceal en el entorno familiar. Cuando las familias no tienen las respuestas, los jóvenes describen distintos modos de enfrentar la situación. Destacan la apertura de sus familiares para buscar soluciones, que van desde contactar dentro del entorno a quienes sepan hasta ofrecer el acceso a internet. Para los jóvenes es un punto diferencial, dado que al navegar se configura la posibilidad de orientarse, de encontrar respuestas. Se evidencian así las facilidades que brindan a las nuevas generaciones las tecnologías cuando se trata de relacionarse con el aprendizaje y emprender búsquedas para los estudios. Pero este no es un movimiento solitario, sino que se da en un acompañar, en el habilitar la exploración. Desde esta perspectiva, es el saber-hacer lo que algunas familias despliegan frente al desconocimiento académico, y ello es importante para los jóvenes, quienes subrayan el sentido de disponibilidad en su entorno, que permite dar respuestas.

Lo expresado por los jóvenes es otra interpretación de las familias en su vínculo con la educación. No es la cara institucional, sino la cara interior, es la que contradice el imaginario social que insinúa que estas familias no apoyan a sus hijos, a sus nietos. En escenas cotidianas, las familias despliegan variadas formas de apoyar a las nuevas generaciones en sus itinerarios educativos. Pero ello no siempre es evidente para las instituciones educativas y sus agentes. Al preguntar y atender a sus respuestas, es posible captar los modos de transmisión y el esfuerzo por acompañar que se defiende en lo cotidiano, en gestos que casi ni se notan, imperceptibles para una mirada ajena; pero a través del ejercicio de recuperación, de detención en diálogos íntimos, se identifica que esos gestos van dejando huellas en estos jóvenes, que les permiten continuar estudiando. Desde esta óptica, comprender cómo se despliegan estos apoyos implica hacer lugar e instalar la palabra. Acarrea la disposición a ver - y a escuchar, en particularcomprender cómo se compensan y recompensan en las familias estas noveles biografías educativas. 


\section{Apuntes finales: sobre la generación de sentidos y los sentidos de las generaciones}

Este artículo se inició caracterizando la educación media en Uruguay en comparación con el escenario actual del sistema educativo. Sobre ello es posible afirmar que el mandato de selección que dio origen al liceo aún se mantiene y contrasta con la propuesta de universalización que se propone para este siglo. En este marco, como indica Núñez (2003), es necesario pensar nuevas maneras de sostener la responsabilidad pública ante las generaciones que llegan, hacer un esfuerzo de invención y tomar una opción política.

El uso de nuevos términos, como universalización, es un avance conceptual que no equivale a la construcción de itinerarios educativos para todas las personas, ya que, si no se apela a la distribución de las desigualdades, si esta se mantiene inalterada e invisible, las nuevas formas de decir quedan sin efecto. Para los jóvenes de Casavalle, las distancias simbólicas también se cristalizan a partir de la dimensión territorial, en cuanto la amplia oferta educativa se da por fuera de su barrio.

Es preciso señalar que hasta comienzos del siglo XXI no existían allí establecimientos de nivel secundario. Es decir, el liceo no fue algo cercano hasta esa fecha. Pero en la actualidad esta situación se ha ido modificando, ya que surgieron diversas propuestas de educación media (Liceos 69 y 73, FPB/ Cy otras ofertas privadas). Igualmente, en el presente no existen propuestas públicas de educación media superior y por tanto es posible establecer que la falta de infraestructura y oferta educativa coadyuva en la continuidad o discontinuidad educativa. En esta línea, Casavalle ha sido un ejemplo paradigmático.

A primera vista la reflexión en torno a ser primera generación es una discusión sobre una categoría social, porque no surge como un aspecto relevante para los jóvenes. Al momento de preguntar sobre ello, era un aspecto no pensado, sin resonancias; parecía un constructo para comprender a otros, en el que los jóvenes no se identificaban. Pero al escuchar, analizar y evocar sus historias, lo generacional se trasluce y aparece como relevo, conflicto o giro generacional. En sus narraciones se expresa la importancia del otro que se encuentra disponible para la transmisión del legado, indicando que el adulto es significativo en su ausencia o presencia. Los jóvenes recuperan los diálogos sobre lo educativo, describen los momentos de crisis y la necesidad de empuje, y sus relatos no son exclusivos del ámbito familiar, sino que incluyen también a docentes y adscriptos con quienes inscriben subjetividad en la escena liceal.

En la historia han existido diversas primeras generaciones, pero en este contexto fue necesario abrir la pregunta sobre esta generación e identificarla desde la noción de lo inédito, de aquello que conduce a lo nuevo. Según Tarrab, es la posibilidad «de que lo 
escrito, pero aún no editado, encuentre una nueva inscripción, de que lo aún no escrito pueda escribirse de un modo nuevo para salir de la repetición» (2001, p. 5). Por tanto, producir lo inédito requiere situarse en nuevas posiciones sin saber lo que acontecerá. En este sentido, lo inédito en la biografía del sujeto provoca una novedad en la trama familiar y la embarca en una experiencia sin precedentes.

El liceo tiene sentido para estos jóvenes, aspecto que coincide con los resultados de Llinás (2009) y Ruiz Barbot (2015), quienes consignan el valor positivo asignado a lo educativo en la actualidad. No obstante, emerge el sentir de que el liceo no es válido para algunos de ellos (por sus formatos, su propuesta curricular, por las distancias simbólicas que representa, por su escasa hospitalidad), lo cual es muy distinto a creer que para ellos no tiene valor. Esta afirmación es posible porque los jóvenes transmiten un afán de futuro, ingresan con curiosidad, inquietos, pero, a medida que estas expectativas no son reflejadas desde la institución, se enfrentan a un contrasentido. Ese ímpetu inicial se despliega en una variedad de itinerarios a medida que transcurre la experiencia: en ciertos jóvenes se mantiene, en algunos se erosiona y en otros se desvanece.

La llegada y sus derroteros no son iguales para todos. En algunos jóvenes la vivencia liceal es aprehensible, logran comprender e identificar vocaciones durante sus itinerarios educativos. Continúan en los liceos y son críticos al expresar las distancias y reconocer los contrastes que experimentan al estudiar fuera de sus barrios. En otros jóvenes es posible identificar el desacople como una parte clave del problema que configura una subjetividad en deuda: aunque conozcan y reconozcan el oficio de ser estudiantes, ellos perciben que en el liceo no hay lugar para ellos: hay una desligazón entre el sujeto y la institución liceal. En las experiencias, y sus vicisitudes, es posible identificar la distinción entre la inscripción simbólica y la administrativa que el liceo realiza, para algunos u otros estudiantes, distinción que los saca de este recorrido al no dejar huella en su pasaje, al no acompañar en la producción de sentido.

Los jóvenes también son categóricos al expresar que cuando no se entiende no se aprende; saben que eso los deja afuera y reivindican el sentido pedagógico de la experiencia liceal. No obstante, en este marco internalizan la responsabilidad del fracaso que se ensambla con otras voces: se «desvincularon», «dejaron», «desertaron», etc. La culpa está privatizada en el sujeto y se encuentran desdibujadas las responsabilidades educativas e institucionales, aspecto coincidente con lo señalado por Dubet y Martuccelli (1996), Frigerio (2002) y Martinis (2006).

Es posible afirmar que la experiencia liceal trasciende al liceo, declaración simple y obvia, pero sustancial, que contradice el enfoque que reduce el liceo a lo curricular y lo distancia de lo social. Ellos componen una variedad de sentidos sobre su experiencia liceal, que son constitutivos y refieren al vínculo con el aprendizaje, los docentes, los pares, y suman otros: sus familias, el futuro y también el barrio. Los sentidos formulados por los jóvenes en torno a ser los «primeros en llegar» coinciden con los resultados 
hallados por Dubet y Martuccelli (1996) y Falconi (2009), en tanto los relatos exponen las ambivalencias del heredar/ desheredar y las contradicciones que emergen cuando no se alcanza a cubrir las expectativas de sus familias. Las narrativas analizadas describen escenas que van del orgullo al desencanto, cuando los estudiantes experimentan la ambivalencia familiar. Sus recorridos educativos tienen efectos subjetivos, que van de la «negación» a la «superación», en las distancias que establecen respecto a sus lugares de pertenencia.

En este contexto se identifica que las familias despliegan operaciones subjetivantes, lo que se aparta de las visiones que señalan que las familias han perdido fuerza para estructurar la personalidad. No se advierten los «modos desubjetivantes» de habitar los vínculos familiares, que se han identificado en otros contextos de mayor vulneración de derechos, como indican Duschatzky y Corea (2002). A través de los relatos y la recuperación de la memoria se identifican gestos que van dejando huellas en estos jóvenes primera generación, que les permiten continuar estudiando. Estas son escenas que en el liceo se desconocen y sin embargo son cotidianas.

La voz de los estudiantes afirma las coincidencias entre los datos, las teorías y sus narrativas y ofrece nuevas perspectivas a un fenómeno que tiene diversos ángulos en la intersección entre lo social, lo familiar y el sujeto. Específicamente, se identifica en los jóvenes que se encuentran con lo distinto en el ámbito liceal una posición que va del comparar al mantener, del mediar al cambiar, y en dicho proceso se resignifica o se sostiene lo heredado. Pero lo sustancial en esta descripción es el recorrido; el movimiento en el sujeto, en la subjetividad. El escenario liceal se ha transformado y requiere acompasarlo con cambios. Por tanto, la discusión sobre las responsabilidades es inevitable, y no se puede deslindar de las opciones de políticas educativas que se configuran y ofrecen. Los jóvenes dan señales de sus requerimientos y la importancia de los otros para llevarlos a cabo. Sus narrativas cuentan sobre los umbrales, sobre la capacidad de los sujetos de producir historias que conducen a los cambios. Nos advierten y dan certeza sobre las formas que la educación debe adquirir y, en especial, evidencian que el porvenir se compone entre generaciones, con la disponibilidad familiar y la tenacidad de las biografías inéditas.

\section{Referencias bibliográficas}

Acosta. F. (2011). Estudio sobre casos de políticas educativas destinadas a la Educación Secundaria. Buenos Aires: IIPE UNESCO.

Aguiar, S. (2009). Acercamientos a la segregación urbana en Montevideo. (Tesis de doctorado). Universidad de la República, Facultad de Ciencias Sociales, Uruguay. 
Álvarez Pedrosian, E. (2013). Casavalle bajo el sol: Investigación etnográfica sobre territorialidad, identidad $y$ memoria en la periferia urbana de principios de milenio. Montevideo: CSIC.

Ardoino, J. (2005). Complejidad y formación: Pensar la educación desde una mirada epistemológica. Buenos Aires: Centro de Publicaciones Educativas y Material Didáctico.

Baquero, R. (2001). La educabilidad bajo sospecha. Cuaderno de Pedagogía, 9, 71-85.

Beck, U. y Beck-Gernsheim, E. (2003). La individualización: El individualismo institucionalizado $y$ sus consecuencias sociales $y$ políticas. Barcelona: Paidós.

Bertaux, D., y Wiame, I. B. (1994). El patrimonio y su linaje: Transmisiones y movilidad social en cinco generaciones. Estudios sobre las Culturas Contemporáneas, 6(18), 27-56.

Bleichmar, S. (2003). Acerca de la subjetividad. Recuperado de http:/ / seminario-rs.gcrosario.com.ar/ conf-silvia-bleichmar-30-07-2003.

Bordoli, E. (2007). La tríada del saber en lo curricular: Apuntes para una teoría de la enseñanza. En L. Behares y C. Blezio (comps.), El borde de lo (in)enseñable. Anotaciones para una teoría de la enseñanza (pp. 27-52). Montevideo: FHCE.

Bourdieu, P. y Passeron, J. C. (1964). Los herederos, los estudiantes y la cultura. Buenos Aires: Siglo XXI.

Cardozo, S. (2016). Trayectorias educativas en la educación media: Pisa-L 2009-2014. Montevideo: INEEd.

Casal,J. (1996). Modos emergentes de transición a la vida adulta en el umbral del siglo XXI: Aproximación sucesiva, precariedad y desestructuración. Reis: Revista Española de Investigaciones Sociológicas, 75, 295-316.

Casal, J., García, M., Merino, R. y Quesada, M. (2006). Aportaciones teóricas y metodológicas a la sociología de la juventud desde la perspectiva de la transición. Papers: Revista de Sociología, 79, 21-48.

Castel, R. (2004). La inseguridad social. ¿Qué es estar protegido? Buenos Aires: Manantial. Contreras-Domingo, J. (2013). El saber de la experiencia en la formación inicial del profesorado. Revista Interuniversitaria de Formación del Profesorado, 78(3), 125-136.

De Armas, G. y Retamoso, A. (2010). La universalización de la educación media. Montevideo: UNICEF.

De Gaulejac, V. (2013). La neurosis de clase. Buenos Aires: Nuevo Extremo.

Delio, L. (2011). Algunas reformas de planes de estudio de enseñanza secundaria durante el período 1908-1935. Anales del Instituto de Profesores Artigas, 5, 71-90.

Denzin, M. y Lincoln, Y. (1994). Handbook of qualitative research. California: Sage.

Di Leo, P. (2011). Escuelas y violencias, desplegando una relación problemática. Revista de la Escuela de Ciencias de la Educación, 6, 231-248. 
Dibot, G. (2015). Tendencia no es destino: jóvenes en programas de inclusión de educación media en Uruguay (Tesis de maestría). Universidad de la República, Facultad de Ciencias Sociales, Montevideo.

Du Bois-Reymond, M. y López Blasco, A. (2004). Transiciones tipo yo-yo y trayectorias fallidas: Hacia las políticas integradas de transición para los jóvenes europeos. Revista de Estudios de Juventud, 65(04), 11-29.

Dubet, F. (2011). Sociología de la experiencia. Madrid: Complutense.

Dubet, F. y Martuccelli, D. (1996). En la escuela: Sociología dela experiencia escolar. Buenos Aires: Losada.

Duschatzky, S. y Corea, C. (2002). Chicos en banda: Los caminos de la subjetividad en el declive de las instituciones. Buenos Aires: Paidós.

Dussel, I. (2009). ¿Qué lugar tiene la escuela media en la producción y reproducción de la desigualdad? Elementos para el debate. Revista de Política Educativa, 1(1), 67-90.

Espíndola, F. (2007). Representaciones sociales de los jóvenes desde los espacios de la exclusión social: De ser joven y vivir en Casavalle sin estudiar ni trabajar. (Tesis de Maestría). Universidad de la República, Facultad de Ciencias Sociales, Montevideo.

Espíndola, F. (2009). De jóvenes «ni, ni» que habitan Casavalle: Representaciones sociales desde espacios de exclusión. Revista de Ciencias Sociales, 25, 93-105.

Falconi, O., Foglino, A. M. y López Molina, E. (2008). Una aproximación a la construcción de la experiencia escolar de adolescentes y jóvenes de grupos sociales urbanos en condiciones de pobreza en Córdoba. Cuadernos de Educación, 6(6), 227-243.

Feixa, C. y Canelles, N. (2007). De bandas latinas a asociaciones juveniles: la experiencia de Barcelona. Educação, 1(61), 11-28.

Fernández, T. (2010). Enfoques para explicar la desafiliación. En T. Fernández (coord.). La desafiliación de la educación media y superior de Uruguay. Montevideo: CSIC.

Filardo, V. y Mancebo, M. E. (2013). Universalizar la educación media en Uruguay: Ausencias, tensiones $y$ desafíos. Montevideo: CSIC.

Filardo, V. (2005). Hacia la resignificación de «Casavalle». Santiago de Chile: CEPAL.

Filardo, V. (2010). Transiciones $a$ la adultez y educación. Montevideo: UNFPA. (Cuadernos del UNFPA, Serie Divulgación, 4, 5).

Flick. U. (2004). Introducción a la investigación cualitativa. Madrid: Morata.

Foglino, A. M., Falconi, O. y López Molina, E. (2009). El desafío de incorporar a los que recién llegan. Experiencia escolar y subjetividad en adolescentes y jóvenes en situación de pobreza. Revista Hoy la Universidad, 2, 11-13.

Folgar, L. y Romano, A. (2007). Discursos, traducciones y construcción de identidades: La escuela como espacio privilegiado de disputa simbólica. En G. Batallán y A. Gomes (coords.), Ciudadanía, exclusión y diversidad sociocultural: Niños $y$ jóvenes en 
contextos de socialización, 7. Reunión de Antropología del Mercosur. Porto Alegre, Brasil.

Frigerio, G. (2002). Entre la repetición y la ocasión de lo nuevo: El derecho a inventar otra cosa. En ¿Qué es la educación secundaria para el siglo XXI? (pp.47-72). Santiago de Chile: Unesco.

Glasser, B. y Strauss, A. (1967). El desarrollo de la teoría fundada. Chicago: Aldine.

Instituto Nacional de Evaluación Educativa. (2019). Informe sobre el estado de la educación en Uruguay 2017-2018. Montevideo: INEEd.

Karsz, S. (coord.) (2004). La exclusión: Bordeando sus fronteras. Definición y matices. Barcelona: Gedisa.

Kessler, G. (2002). La experiencia escolar fragmentada: Estudiantes $y$ docentes en la escuela media en Buenos Aires. Buenos Aires: IIPE- UNESCO.

Kessler, G. (2007). Juventud rural en América Latina: Panorama de las investigaciones actuales. En R. Bruniard (coord.), Educación, desarrollo rural y juventud. Buenos Aires: IIPE-UNESCO.

Ley 18437 (2008). Ley General de Educación. Recuperado de https://www.ineed. edu.uy/ images/ pdf/-18437-ley-general-de-educacion.pdf.

Llinás, P. (2009). Imágenes y sentidos de la experiencia escolar: Percepciones de los estudiantes sobre la escuela secundaria. Propuesta Educativa, 32, 95-104.

Lombardo, C. (2005). Hacia la resignificación de Casavalle, Montevideo, Uruguay: lineamientos físico-territoriales. Santiago de Chile: CEPAL.

Mancebo, M. E. y Goyeneche, G. (2010). Las políticas de la inclusión educativa: Entre la exclusión social y la innovación pedagógica. En 9.as Jornadas de investigación de la Facultad de Ciencias Sociales. Montevideo, Uruguay. Recuperado de http:// www.fcs.edu.uy/ archivos/Mesa_12_y_17_Mancebo-Goyeneche.pdf

Mancebo, M. E., y Monteiro, L. (2009). El programa de aulas comunitarias de Uruguay: un puente hacia la inclusión en la Educación Media. REICE: Revista Electrónica Iberoamericana sobre Calidad, Eficacia y Cambio en Educación, 7(4), 278-291.

Martinis, P. (2006). Educación, pobreza e igualdad: Del «niño carente» al «sujeto de la educación». En P. Martinis y P. Redondo (comps.), Igualdad y educación: Escritura entre (dos) orillas (pp. 13-32). Buenos Aires: Del Estante.

Martinis, P. (2013). Educación, pobreza y seguridad en el Uruguay de la década de los noventa. Montevideo: Ediciones Universitarias.

Martinis, P., Míguez, M. N., Viscardi, N. y Cristóforo, A. (coords.). (2016). Derecho a la educación $y$ mandato de obligatoriedad en la enseñanza media: La igualdad en cuestión. Montevideo: CSIC.

Merklen, D. (2005). Pobres ciudadanos: Las clases populares en la era democrática (Argentina 1983-2003). Buenos Aires: Gorla. 
Núñez, P. (2010). Política y poder en la escuela media: Disputas en torno a la «participación juvenil» en el espacio escolar. Revista Argentina de Estudios de Juventud, $1(2)$.

Núñez, V. (2003). Los nuevos sentidos de la tarea de enseñar: Más allá de la dicotomía «enseñar vs asistir». Revista Iberoamericana de Educación, 33, 17-35.

Núñez, V., (1999). Pedagogía Social: Cartas para navegar en el nuevo milenio. Buenos Aires: Santillana.

Pla, M. (1999). El rigor en la investigación cualitativa. Atención Primaria, 24(5), 295300.

Romero, P. (2018). Generación inédita: Sentidos de la experiencia liceal en jóvenes de Casavalle, que continúan estudiando y son primera generación en educación media. (Tesis de maestría). Universidad de la República, Facultad de Psicología. Montevideo. Recuperado de https:/ / hdl.handle.net/ 20.500.12008/ 20051.

Ruiz Barbot, M. (2015). Los sentidos de la educación en adolescentes, familias y referentes comunitarios: Informe final. Montevideo: MEC.

Ruiz Barbot, M, Barceló, J., Gatti, E., Maestro, C., Píriz, S. y Weisz, B. (2014). Una aproximación $a$ las demandas de los actores sociales externos al sistema educativo formal, respecto $a$ la educación. Informe final. Montevideo: INEEd.

Saraví, G. (2009). Transiciones vulnerables: Juventud, desigualdad y exclusión en México. México: CIESAS.

Skliar, C. (2011). Fragmentos de experiencia y alteridad. En J.Larrosa y C. Skliar (comp.), Experiencia y alteridad en educación. Rosario: Homo Sapiens.

Stauber, B. y Walthers, A. (2001). Avoiding misleading trayectories: Transition dilemmas of young adults in Europe. Recuperado de www.nuffield.ox.ac.uk/projects/ uwwclus/ Papers/ restrict/ misleading.pdf.

Tarrab, M. (2001). Un lazo social inédito. Virtualia: Revista digital de la Escuela de la Orientación Lacaniana, 2, 2-14.

Tenti Fanfani, E. T. (2007). La escuela y la cuestión social: Ensayos de sociología de la educación. Buenos Aires: Siglo XXI.

Terigi, F. (2009). Las trayectorias escolares: Del problema individual al desafío de política educativa. Buenos Aires: OEA.

Tiramonti, G. (2011). Variaciones sobre la forma escolar: Límites y posibilidades de la escuela media. Rosario: Homo Sapiens.

Tiramonti, G., Arroyo, M., Nobile, M., Montes, N., Poliak, N., Sendón, M. y Ziegler, S. (2008). Una experiencia de cambio en el formato de la escuela media: Las escuelas de reingreso de la Ciudad de Buenos Aires. Propuesta Educativa, 17(30), 57-69.

Urresti, M. (2000). Cambio de escenarios, experiencia juvenil urbana y escuela. En E. Tenti Fanfani (comp.), Una escuela para los adolescentes. Buenos Aires: Losada. 
Vaccotti, R. (2014). La relación familia-institución educativa en enseñanza media. Perspectivas de docentes de secundaria. (Tesis de maestría). Universidad Católica, Facultad de Psicología. Montevideo, Uruguay.

Ventós Coll, M. (2015). ¿Por qué continúan los que continúan en educación media básica? Sentidos que se atribuyen a los jóvenes que retoman o permanecen en sus estudios a través del Plan de Formación Profesional Básica 2007. (Tesis de maestría). Universidad de la República, Facultad de Psicología. Montevideo, Uruguay.

Viscardi, N. y Alonso, N. (2013). Gramática(s) de la convivencia: Un examen a la cotidianidad escolar $y$ la cultura política en la educación primaria y media en Uruguay. Montevideo: ANEP.

Wacquant, L. (2001). Parias urbanos: Marginalidad en la ciudad $a$ comienzos del milenio. Buenos Aires: Manantial.

Wacquant, L. (2010). Las dos caras de un gueto: Ensayos sobre marginalización y penalización. Buenos Aires: Siglo XXI.

Weiss, E. (2012). La educación media superior en México ante el reto de su universalización. Archivos de Ciencias de la Educación, 6(6). Recuperado de https:/ / www.archivosdeciencias.fahce.unlp.edu.ar.

Zelmanovich, P. y Minnicelli, M. (2012). Instituciones de infancia y prácticas profesionales: entre figuras de segregación y dispositivos de inscripción simbólica. Propuesta Educativa, 37, 39-50. 